\title{
A FIBER OPTIC MICROWAVE POWER PROBE
}

\author{
V.M. MARTIN, Asst. Prof. of Physics and \\ S.K. ANGELL, AsSOC. Prof. of Electrical Engineering, \\ USAF Academy, Colorado Springs, Co 80840
}

R.M. SEGA, Asst. Prof. of Electrical Engineering, University of Colorado, Colorado Springs, CO 80907

\section{Introduction}

All measurements, scientific or otherwise, are always subject to errors resulting from the interaction of the measurement device with the quantity being measured. When dealing with microwaves, the fields are typically detected with some type of antenna which must be electrically connected to a recording instrument. The antenna as well as its electrical connection can, at times, significantly perturb the field being measured. By using fiber optics to the maximum extent in our detectors, microwave fields may be perturbed only minimally since the entire probe and associated connections are dielectric. This paper presents a new microwave measurement technique that employs a commercially available fiber optic digital thermometer built by Luxtron Corporation. In this measurenent scheme a small amount of slightly conductive material is applied to the fiber optic sensor. When the coated sensor is placed in a microwave field, currents are induced in the coating which in turn produces joule heating. We show experimentally that under certain circumstances the coating temperature is linearly related to the power level present.

\section{Theory}

Given a finite sized body, the total power absorbed, $P$, would be given by 1

$$
P=\sigma E_{i}^{2} d V
$$

where $\mathrm{V}$ is the volume of the body, $\sigma$ is the conductivity, and $E_{j}$ is the electric field internal to the body. Since $\sigma$ is not a function of temperature (It actualiy varied less than 0.58 over the temperature range studied. ${ }^{2}$, , there are no material changes of state, and power is deposited only via joule heating then we may write $P=C P$ incident where $C$ is a constant. 3 Now if we consider the one-dimensional, steadystate solution to the heat equation we have ${ }^{2}$

$$
\frac{\Delta Q}{\Delta t}=K \frac{\Delta T}{l} A
$$

U.S. Government work not protected by U.S. copyright. 
where $\frac{\Delta Q}{\Delta t}$ is the power conducted through a slab of thickness $\ell$, area $A$, constant thermal conductivity $K$, and temperature gradient across $l$ of $\Delta T$. This would be valid if the thickness of $\ell$ is very small compared to the lateral dimensions of a material. By insulating a small conductive probe with foam and further assuming the external surface temperature is approximately that of ambient air, we have constructed a power probe linear in $\Delta T$. We measure the probe temperature with a fiber-optic thermometer; hence, $\Delta \mathrm{T}=\mathrm{T}_{\text {probe }} \mathrm{T}_{\mathrm{T}} \mathrm{ix}$. In other words, we have

$$
C P_{\text {inc }}=\mathrm{K} \frac{\Delta \mathrm{T}}{\ell} \mathrm{A}
$$

for the quilibrium condition. Simplifying, we have

$$
P_{\text {incident }}=B \Delta T
$$

where $B \equiv K A / C l$, thus showing that temperature rise in the probe is linearly related to microwave power density. $B$ will be determined empirically since it will be a function of thermodynamic as well as electrical characteristics and is strongly frequency dependent.

\section{Design and Fabrication}

An example probe design that incorporates some of the above properties is illustrated in Figure 1 below.

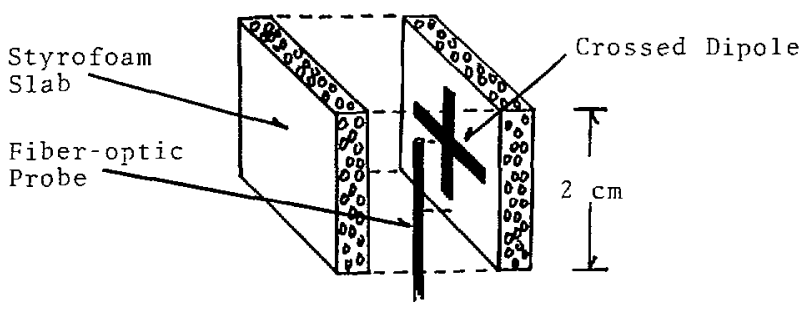

Figure 1: Typical Probe Design.

The insulator was cut from styrofoam with a measured relative permittivity of 1.1 at $10 \mathrm{GHz}$. The crossed dipole was made from carbon based spray paint known as Television Tube Coat. It has a measured conductivity of $315 \mathrm{mhos} / \mathrm{m}^{2}$ and was layered until a thickness of approximately $50 \mu \mathrm{m}$ was reached.

\section{Experiment}

Experiments were conducted to determine the linearity, sensitivity, and time response of the device. 
Linearity was determined by measuring $\Delta T$ at power densities from 0 to $40 \mathrm{~mW} / \mathrm{cm}^{2}$. See Figure 2 below.

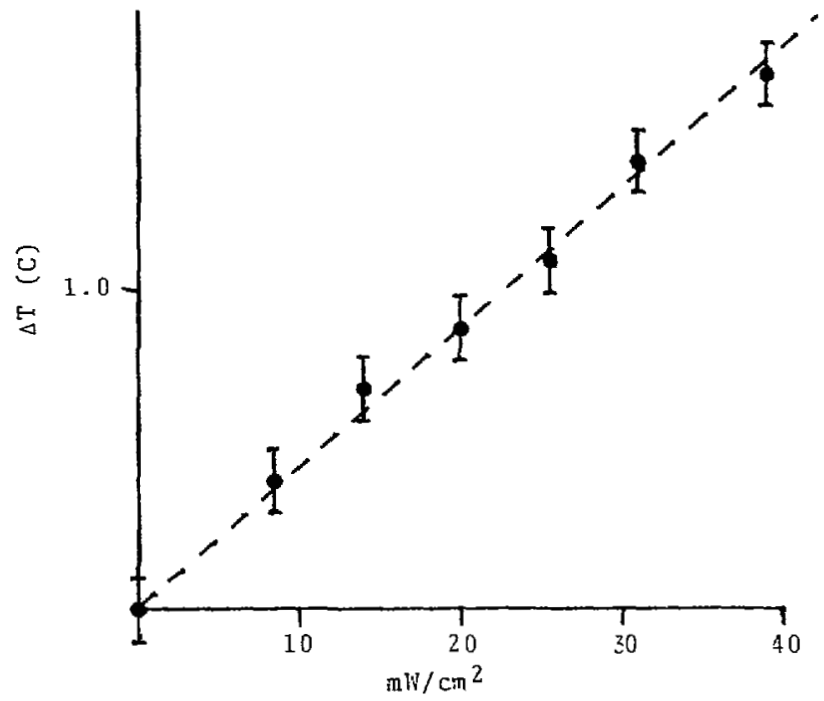

Figure 2: Probe Iinearity; Power Density vs. $\Delta \mathrm{T}$.

Figure 3 is a plot of system noise. It can be seen that a maximum error of $\pm .1 \mathrm{C}$ could result from these variations. It is felt that the large fluctuations result from room temperature variations and could possibly be reduced with a continuous room temperature feedback system. See Figure 3 below.

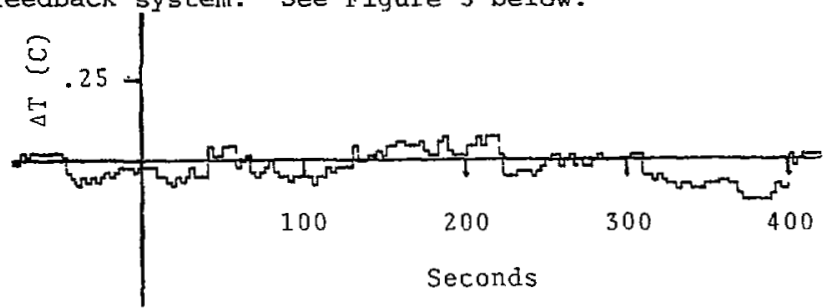

Figure 3: System Noise.

Lastly, the time response is illustrated in Figure 4. It may be seen that for our particular probe configuration we have a response time of approximately 2 minutes. (Time to reach 998 of maximum.) see Figure 4 
below.

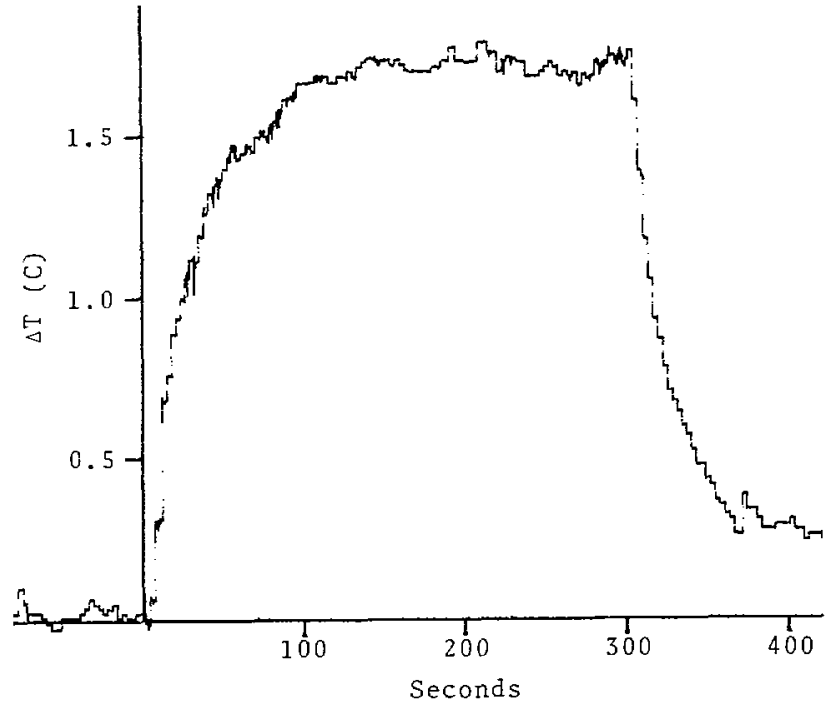

Figure 4: Time Response; $39 \mathrm{~mW} / \mathrm{cm}^{2}$ Applied From $t=0$ s to $t=300 \mathrm{~s}$.

\section{Conclusions}

This technique may be useful for microwave power density measurements; however, it has a large time response, is effective only for relatively large power densities and may be subject to significant errors. Its biggest advantage is that it is relatively noninteractive and is straight forward to use. The errors might well be reduced by a magnitude of 10 or 100 if a second system were used to measure the ambient air temperatures near the primary probe and thus provide continuous feedback for $\Delta \mathbf{T}$ calculations.

\section{Bibliography}

1. E.C. Jordan and K.G. Balmain; Electromagnetic Waves and Radiating Systems, 1968; Prentice Hall; Englewood Cliffs, NJ.

2. V.M. Martin; Electromagnetic Interaction with Thin Multiple Conductive Coatings; 1982; University of Colorado Dissertation. 3. V.A. Fock; Electromagnetic Diffraction and Propagation Problems; 1965; Pergamon Press; New York. 